\title{
MAGIC Follow-up of Gravitational Wave Events in the Third LIGO/Virgo Observing Run
}

\section{A. Berti ${ }^{* 1}$, M. Ribó ${ }^{2}$, L.A. Antonelli ${ }^{3}$, J. Becerra González ${ }^{4}$, Ž. Bošnjak ${ }^{5}$, S. Covino ${ }^{6}$, B. De Lotto ${ }^{7}$, F. Del Puppo ${ }^{8}$, S. Inoue ${ }^{9}$, F. Longo ${ }^{8}$, D. Miceli ${ }^{7}$, E. Moretti ${ }^{10}$, L. Nava ${ }^{6}$, B. Patricelli ${ }^{11}$, A. Stamerra ${ }^{3}$ for the MAGIC Collaboration}

${ }^{1}$ University of Torino and INFN Torino, Torino, Italy

${ }^{2}$ Universitat de Barcelona, ICCUB, IEEC-UB, Barcelona, Spain

${ }^{3}$ INAF, Rome, Italy, ${ }^{4}$ IAC and Universidad de La Laguna, Tenerife, Spain

${ }^{5}$ Faculty of Electrical Engineering and Computing, University of Zagreb, Zagreb, Croatia

${ }^{6}$ INAF, Osservatorio Astronomico di Brera, Merate, Italy

${ }^{7}$ University of Udine and INFN Trieste, Udine, Italy

${ }^{8}$ University and INFN Trieste, Trieste, Italy

${ }^{9}$ RIKEN, Wako, Saitama, Japan, ${ }^{10}$ IFAE-BIST, Bellaterra (Barcelona), Spain

${ }^{11}$ University and INFN Pisa, Pisa, Italy

E-mail: Alessio.Berti@to.infn.it

The search for gravitational wave (GW) signals has been very successful in the first two observing runs (O1 and $\mathrm{O} 2)$ of the LIGO and Virgo interferometers. In total, eleven GW events were detected [1]: ten from the coalescence of binary black hole systems and one from a binary neutron star system (GW170817) [2]. The latter in particular is the perfect example of the multi-messenger approach in astrophysics, thanks to the detection of an electromagnetic (EM) counterpart as a short gamma-ray burst, GRB170817A [3]. The EM follow-up performed by instruments at different wavelengths is crucial to identify the counterpart, the host galaxy, the nature of the remnant and the properties of its environment. Imaging Atmospheric Cherenkov Telescopes (IACTs) join the follow-up effort searching for very-high energy emission (E>100 $\mathrm{GeV}$ ). The relatively narrow field of view, few square degrees, of IACTs poses a challenge in the detection of the counterpart in the large uncertainty region provided by the interferometers. A well designed and optimised observational strategy is therefore needed to maximize the probability to select the region hosting the counterpart. Among the IACT experiments, the MAGIC collaboration joined the EM follow-up community in 2014, performing the first follow-up of a GW candidate event (GW151226 [4]) by a Cherenkov Telescope. For the current observing run (O3), MAGIC is optimising the GW follow-up strategy taking into account the information provided with the GW alert, the observational constraints, and taking advantage of its fast repositioning system adopted for Gamma-Ray Bursts (GRBs). In this contribution we will present the MAGIC follow-up strategy of GW events, showing different observational approaches depending on the information available from interferometers and EM instruments.

The New Era of Multi-Messenger Astrophysics - Asterics2019

25 - 29 March, 2019

Groningen, The Netherlands

* Speaker. 


\section{Introduction}

The aim of multi-messenger astrophysics is to observe and interpret the data coming from different observatories and particle messengers. The information which can be obtained with this approach can help to solve long-standing problems like the origin of cosmic-rays or the sources of high energy neutrinos. In the last years, the era of multi-messenger science started thanks to the operations of neutrino and gravitational waves (GW) detectors like IceCube and LIGO/Virgo. In this contribution we focus on the synergy between GW interferometers and Imaging Atmospheric Cherenkov Telescopes (IACTs), detecting gamma rays in the very-high-energy (VHE, $E \gtrsim 100 \mathrm{GeV}$ ) range. In particular, the Major Atmospheric Gamma Imaging Cherenkov (MAGIC) IACT system is well suited to perform the follow-up of GW alerts, thanks to its performance and dedicated strategy for such events.

\section{The MAGIC Telescopes}

MAGIC is a system of two Cherenkov telescopes of $17 \mathrm{~m}$ diameter each, located in the Canary Island of La Palma within the Observatorio Roque de Los Muchachos (ORM) at 2200 meters a.s.l. They detect gamma-ray sources in the VHE band using the Imaging Atmospheric Cherenkov technique [5].

The main figures of merit of the MAGIC telescopes are [6]:

- a low energy threshold, about $50 \mathrm{GeV}$ at trigger level at zenith

- a high sensitivity at the level of $0.7 \%$ of the Crab Nebula flux above $220 \mathrm{GeV}$ in 50 hours

- a lightweight structure to perform fast follow-up of e.g. Gamma-Ray Bursts (GRBs)

- possibility to observe also with moonlight, thanks to specific hardware configurations, with a good sensitivity [7].

MAGIC covers many science cases with its observations: among them, the follow-up of transient sources is one of the main scientific projects, including the observation of GRBs, neutrino alerts, Fast Radio Bursts (FRBs) and the possible counterparts of GW events. Given the small field of view $\left(3.5^{\circ}\right)$ of MAGIC, the follow-up of transient sources relies on external triggers. These alerts are generated by large field of view instruments or ground observatories (for neutrino and GW events) and distributed to interested partners through the GCN (Gamma-ray Coordinates Network) system. The Automatic Alert System (AAS) of the MAGIC telescopes is responsible of receiving and processing the alerts coming through the GCN.

\subsection{The MAGIC Automatic Alert System}

The MAGIC Automatic Alert System (AAS) is a multi-threaded program communicating with the GCN and the Central Control software of the MAGIC telescopes. A schematic diagram of its workflow is shown in Figure 1. The main tasks of the MAGIC AAS are the following:

- receive and validate the alerts coming from the GCN 


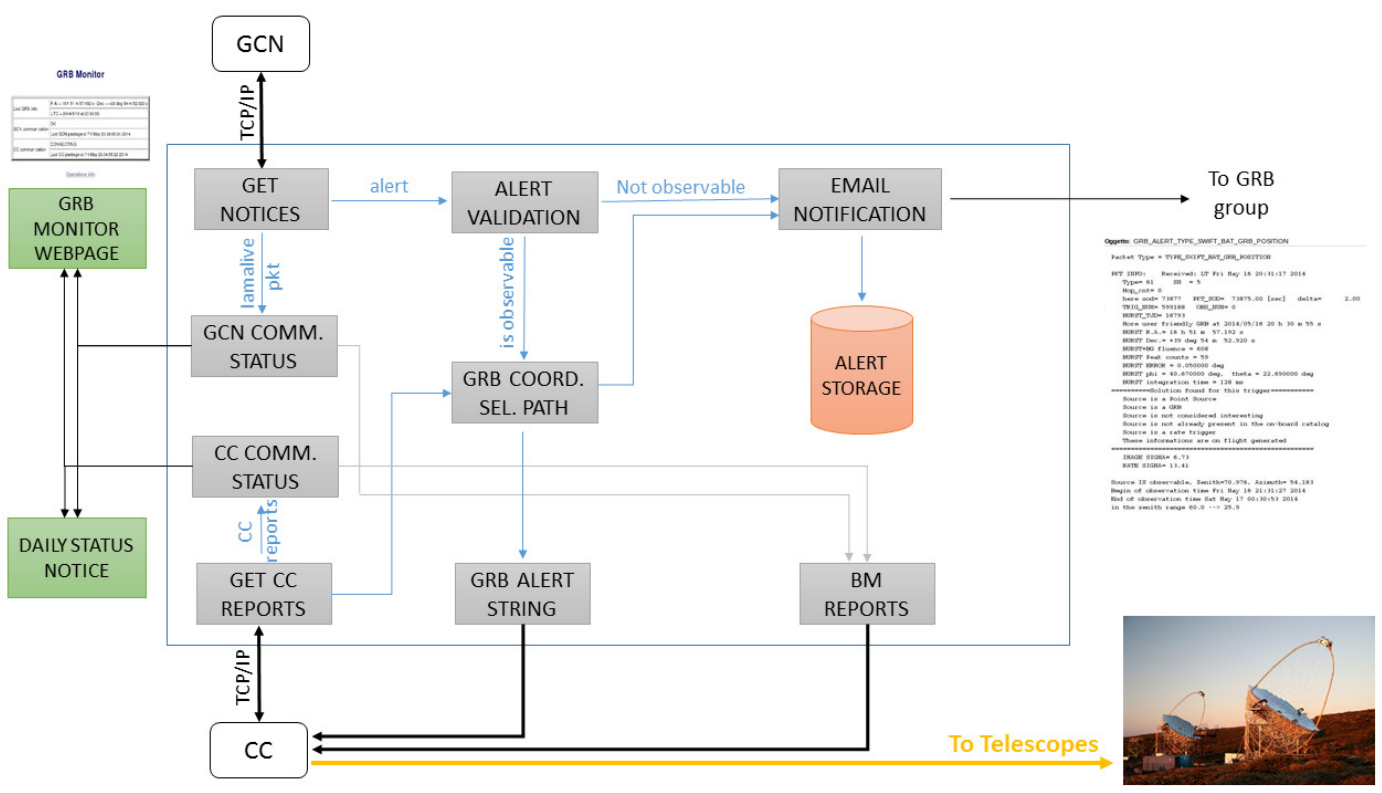

Figure 1: Schematic diagram showing the workflow of the MAGIC Automatic Alert System. Credit: A. Carosi

- decode the content of the alerts, in particular the coordinates of the target if available

- check the visibility of the target from the MAGIC site, according to predefined criteria

- if the target is observable, start the automatic repointing procedure.

The automatic procedure takes care of slewing the telescopes to the target position in fast mode (average speed is $\sim 7 \mathrm{deg} / \mathrm{s}$ ) and preparing the relevant subsystems for data taking. The previous workflow is currently used for GRB and neutrino alerts. For GW alerts coming from LIGO and Virgo, this approach must be changed because of the different information provided in the alerts [9]. In particular, no specific sky position is contained within the alert packet: instead, the URL to an all-sky map is given. An example of such a map is shown in the left panel of Figure 2. For each pixel of the skymap, the probability value that the GW signal comes from that particular direction is contained. Moreover, 3D information about the distance distribution is provided.

This skymap is the starting point of the GW follow-up procedure implemented in the MAGIC AAS.

\section{GW Follow-up Procedure within MAGIC}

As proven in the second observational run of LIGO/Virgo (O2), the follow-up of GW alerts can lead to the detection of the EM counterpart [2,3]. The multi-messenger information is therefore used for the physical characterization of the progenitor system. The possibility of VHE emission in some models [10] makes the follow-up by MAGIC a high-ranked scientific project. For the next LIGO/Virgo observing run (O3), since the number of alerts with good localizations is expected to be higher than in $\mathrm{O} 1$ and $\mathrm{O} 2$ [11], a proper (semi-)automatic observational strategy is needed.

The MAGIC follow-up strategy for GW alerts is foreseen to have different observational cases: 

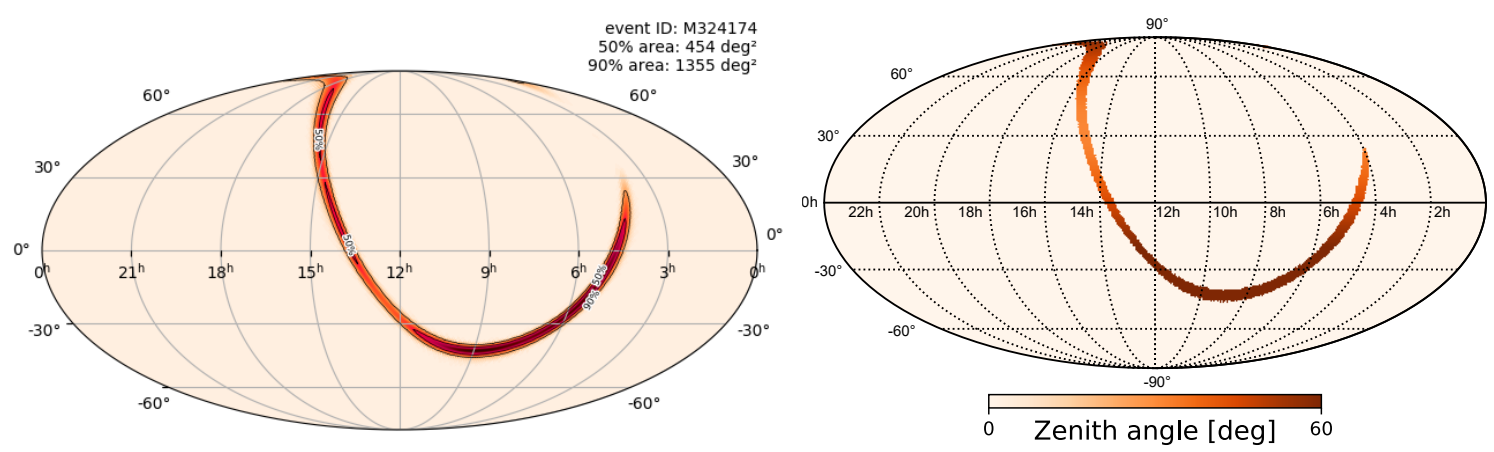

Figure 2: Example of a skymap (left) and the visibility computation from the MAGIC site (right).

1. Autonomous follow-up. For those skymaps whose localization is quite accurate (few tens of square degrees at $90 \% \mathrm{CL}$ ), the strategy will have the following steps:

- compute the visibility of the sky positions from the MAGIC site at different times. An example of such a computation for a GW test skymap is shown in Figure 2.

- for those sky locations with good visibility, provide an ordered list of pointings. The order is given taking into account the galaxy catalogs [12]. An alternative approach is given in [13], which optimises the sequence and the duration of observations maximising both the probability of a detection in a single snapshot and the coverage of the uncertainty region. Using one or the other approach depends much on the distance of the object and on the completeness of galaxy catalogs.

- once the pointing list is defined, the telescopes will be repositioned to the targets position using the automatic procedure through the AAS.

2. Follow-up of identified transients. This observational case falls in the standard automatic follow-up procedure if the putative EM counterparts are distributed through GCN alerts. Otherwise, if communicated through other channels (ATels, GCN Circulars), the observation of such counterparts will be scheduled accordingly.

3. Observation of delayed emission. In the case an off-axis GRB is associated to the GW signal, a delayed EM emission on timescales of weeks/months is expected, as happened in the case of GW170817/GRB 170817A [14, 15]. The scheduling of such observations will be carried out as soon as EM partners share information about the possible delayed emission.

Due to the limited duty cycle of an IACT like MAGIC, for each GW event, depending on the information available, the benefit of performing the follow-up is evaluated. In the case of a confirmed EM counterpart, these observations have highest priority over other targets and so they should be performed as soon as possible. In the opposite case, when no EM counterpart is found, priority is given to the observations related to the other scientific projects.

\section{Conclusion and outlook}

The current LIGO/Virgo observational run (O3), thanks to the improved sensitivity of the instruments, will provide many more GW events with better localization than before. The MAGIC 
collaboration is working with great effort to refine the follow-up strategy for GW events in order to possibly detect the EM counterparts. This will ensure the observation of the most promising candidate events close to their onset, maximizing the chances of detection of GRB-like counterparts, or weeks/months later searching for VHE delayed emission.

\section{References}

[1] Abbott, B. P. et al. 2018, arXiv:1811.12907

[2] Abbott, B. P. et al. 2017, Phys Rev. Lett., 119, 161101

[3] Goldstein, A. et al. 2017, ApJL, 848

[4] De Lotto, B. et al. 2016, Proc. IAU Symposium 324, 287

[5] Aleksic, J. et al., trial, Astroparticle Physics 72, (2016) 61

[6] Aleksic, J. et al. 2016, Astroparticle Physics 72, 76

[7] Ahnen, M. L. et al. 2017, Astroparticle Physics, 94, 29

[8] Grace DB Web Site: https://gracedb.ligo.org/

[9] LSC Open Alerts Web Site:

https://emfollow.docs.ligo.org/userguide/content.html

[10] Murase, K. et al. 2017, arXiv:1710.10757

[11] Abbott, B. P. et al. 2018, Living Rev. Relativity, 21, 3

[12] Singer, L. P. et al. 2016, ApJS, 226, 10

[13] Patricelli, B. et al. 2018, JCAP, 5, 056

[14] Ruan, J. J. et al. 2018, ApJL 853, L4

[15] D’Avanzo, P. et al. 2018, $A \& A$ 613, L1 